\title{
Parameters influencing the droplet formation in a focusing microfluidic channel
}

\author{
Emil Grigorov ${ }^{1, *}$, Jordan A. Denev ${ }^{2}$, Boris Kirov ${ }^{3}$ and Vassil Galabov ${ }^{3}$ \\ ${ }^{1}$ Faculty of German Engineering Education and Industrial Management (FDIBA), Technical \\ University of Sofia, "Sveti Kliment Ohridski" 8, 1756, Bulgaria \\ ${ }^{2}$ Steinbuch Centre for Computing, Karlsruhe Institute of Technology, Hermann-von-Helmholtz- \\ Platz 1, 76344 Eggenstein-Leopoldshafen, Germany \\ ${ }^{3}$ Department of Industrial Automation, Technical University of Sofia, 1756 Sofia, Bulgaria
}

\begin{abstract}
In the present work a detailed numerical study of the parameters influencing the droplet formation in a flow-focusing microfluidic device are made. First, an extensive verification of the simulations with data from the literature is carried out. Influence of parameters like viscosity and inflow velocity are compared with the results from literature showing a good agreement. Some differences are attributed to the different numerical techniques used: in the present work a pure volume-of-fluid method is used, while in the reference study this method is combined with the level-set method. As a second step of the verification of the present model, a comparison with experimental data from the literature was carried out which shows a very good agreement. After the verification was completed, eight new simulations are carried out covering a wide range of velocities of the continuous phase $u_{c}$. In these simulations the velocity of the discrete phase $u d$ remains unchanged. The variation of the continuous phase velocity reveals that with increasing the value of $u_{c}$, respectively the value of the capillary number $\mathrm{Ca}$, the droplet length reaches a point of saturation, i.e. a point where the droplet length does not decrease any more. For the present setup this saturation occurs for $\mathrm{Ca}>$ 0,03 . On the other hand, when the velocity of the continuous phase goes towards very low values ( $\mathrm{Ca}<0,01$ for the present setup), the droplet size increases significantly. Further, it was found that for increasing capillary numbers $\mathrm{Ca}$ above a value around 0,015 for water/oil and above 0,025 for water $+40 \%$ glycerol / oil systems, a transmission from the dripping towards the jetting regimes of droplet formation occurs. It was shown that when the viscosity of the continuous phase increases, higher total pressure jumps in the droplet occur, also leading to the formation of smaller droplets.
\end{abstract}

\section{Introduction}

*Corresponding author: egrigorov@,fdiba.tu-sofia.bg 
In recent years, microfluidic devices have emerged as a novel tool for the realization of different biological, chemical and medical processes. The decreased reagents volumes, usually in the nano/picolitres range, can significantly reduce reaction times, costs and energy consumption for certain processes [1]. Droplet-based microfluidics is a novel technique, which allows the encapsulation of different chemical or biological compounds into single picolitre-droplets. This makes it possible for the isolation and control of individual reactions, protecting them from their surroundings and unwanted interactions with other components. These small microreactors have allowed an easily implementable and relatively cheap approach for a broad range of processes including cell lysis [2], DNA purification [3], polymerase chain reaction (PCR) [4] and other. Therefore, understanding the mechanisms behind droplet generation is crucial for the control and efficiency of a droplet-based microfluidic system.

Droplets formation usually occurs when two immiscible liquids intersect each other. The process is achieved either by active (using electric fields) or by passive (using pressuredriven flow and channel geometry) methods. The specific design of the microfluidic channels makes it possible for a dispersed phase, usually water, to be sheared by another continuous phase (hydrocarbon oils, fluorocarbon oils etc. [5]) and to produce uniformsized drops. Different geometry designs are possible, the most frequently used in the praxis are shown in Figure 1: T-junction [6-7] (a), flow-focusing [8-9] (b), and co-flowing [10] (c). The present work studies devices working on the flow-focusing principle.

(a)

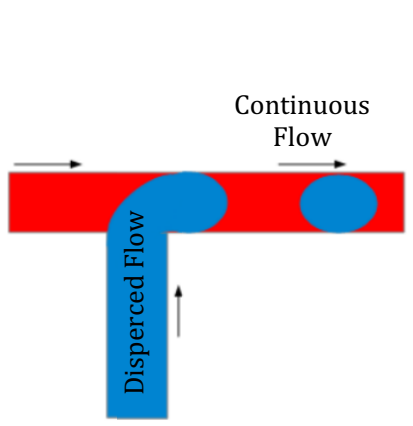

(b)

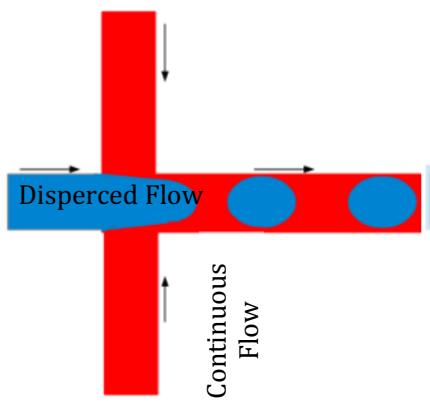

(c)

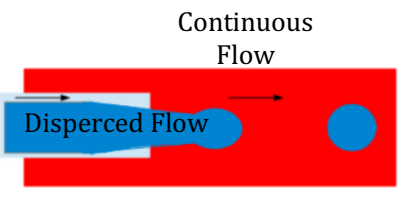

Fig 1. Different geometrical designs for the droplet formation in microfluidic channels : (a) Flowfocusing, (b) T-Junction, (c) Co-flow.

Prior knowledge of droplet size, shape, formation frequency or pressure drop are usually required for the work with devices based on droplet-based microfluidic. Several experimental studies have already investigated the mechanisms behind droplet generation in microfluidic channels [11-15]. Those measurements give an adequate information about the needs of a certain experimental setup; it is not possible however, to include the influence of all effective parameters on the system. A feasible way to collect in advanced the needed information is by utilizing a predictive CFD model. A couple of numerical studies on droplet-based microfluidics were carried out in the past few years making use of different numerical methods (level set (LS) [16], Lattice Boltzmann method (LBM) [17-18] and other).

In this article the droplet formation in a flow-focusing microfluidic channel is investigated numerically by utilizing the volume of fluid (VOF) method. First, we validate our results with data from numerical and experimental studies from the literature. In a second part, using the same geometry as in [19], a parameter study is carried out. It consists of eight 
new velocities of the continuous phase while at the same time the velocity of the dispersed phase was kept unchanged. Thus, the critical capillary number $\mathrm{Ca}$, where the transition between the dripping and the jetting regimes occurs, can be determined. The pressure variation along the center line of the channel for the two flow regimes, is presented and discussed.

\section{Mathematical model, geometrical setup, boundary conditions and numerical details}

In the present study three-dimensional simulations of droplet formation in a flow-focusing geometry as shown in Fig. 2 are carried out. The two immiscible fluids, water and oil as well as their interface, are modelled by the Volume of fluid (VOF) technique. In this Eulerian method, the fluid flow is treated as a continuum. The phase fraction parameter, $\alpha$, indicates the presence of each phase in every control volume of the domain. The surface tension force is distributed near the interface as a body force in the Navier-Stokes equations.

The system of coupled partial differential equation consists of the continuity equation (Eqn.1) the momentum balance equation (Eqn. 2), and the phase fraction equation for $\alpha$ (Eqn. 3) becomes:

$$
\begin{gathered}
\frac{\partial \rho}{\partial \mathrm{t}}+\nabla \cdot(\rho \mathbf{U})=0 \\
\frac{\partial(\rho \mathbf{U})}{\partial \mathrm{t}}+\nabla \cdot(\rho \mathbf{U U})=-\nabla p+\nabla \cdot\left(\mu\left[\nabla \mathbf{U}+\nabla \mathbf{U}^{\mathrm{T}}\right]\right)+\mathbf{F}_{\mathbf{s}} \\
\frac{\partial(\rho \alpha)}{\partial t}+\nabla \cdot(\rho \alpha \mathbf{U})=0
\end{gathered}
$$

In the equations above, $\mathbf{U}$ is the velocity vector field and $\mathrm{p}$ is the pressure field. $\mathbf{F}_{\mathbf{S}}$ represents the surface tension force and is defined as shown in Eqn. 4 and is nonzero only at the interface between the two phases:

$$
\boldsymbol{F}_{\boldsymbol{s}}=\sigma \kappa(\nabla \alpha)
$$

Here $\kappa$ represents the curvature $(\kappa=\nabla .(\nabla \alpha /|\nabla \alpha|))$. Only one such transport equation (Eqn. 3) is solved since the volume fraction of the other phase can be inferred from the limitation:

$$
\alpha_{c}+\alpha_{d}=1
$$

where the index ' $c$ ' stands for continuous and ' $d$ ' for dispersed phase. The viscosity $\mu$ and the density $\rho$ are based on the weighted average of the phase fraction:

$$
\begin{aligned}
& \mu=\alpha \mu_{C}+(1-\alpha) \mu_{\mathrm{d}} \\
& \rho=\alpha \rho_{C}+(1-\alpha) \rho_{\mathrm{d}}
\end{aligned}
$$

In order to verify the results from the VOF method, a geometry of a flow-focusing microchannel, already investigated by Sontti and Atta [19] is considered. The lengths and dimensions of the square cross-section, the inlet and outlet channels are presented in Figure 2 . 
The continuous phase (water/water $+40 \%$ glycerol) is introduced through the two side channels and the dispersed phase (oil: octane $+2,5 \%$ SPAN 80 ) is entered from the main (central) channel. Table 1 summarizes the physical properties of the two oil-water systems considered for the verification of the model and the later calculations in our work. As in [19], the information is obtained from the experimental results of Yao et al. [20].

Table 1. Physical Properties of Continues and Dispersed Phases.

\begin{tabular}{|c|c|c|}
\hline Fluid & Density $-\boldsymbol{\rho}\left[\mathbf{k g} / \mathbf{m}^{\mathbf{3}}\right]$ & Viscosity $-\boldsymbol{\mu}[\mathbf{m P a s}]$ \\
\hline Water & 995,4 & 0,89 \\
\hline Water $+40 \%$ Glycerol & 1090,4 & 3,32 \\
\hline Oil (Octane $2,5+5 \%$ span 80$)$ & 689,9 & 0,53 \\
\hline
\end{tabular}

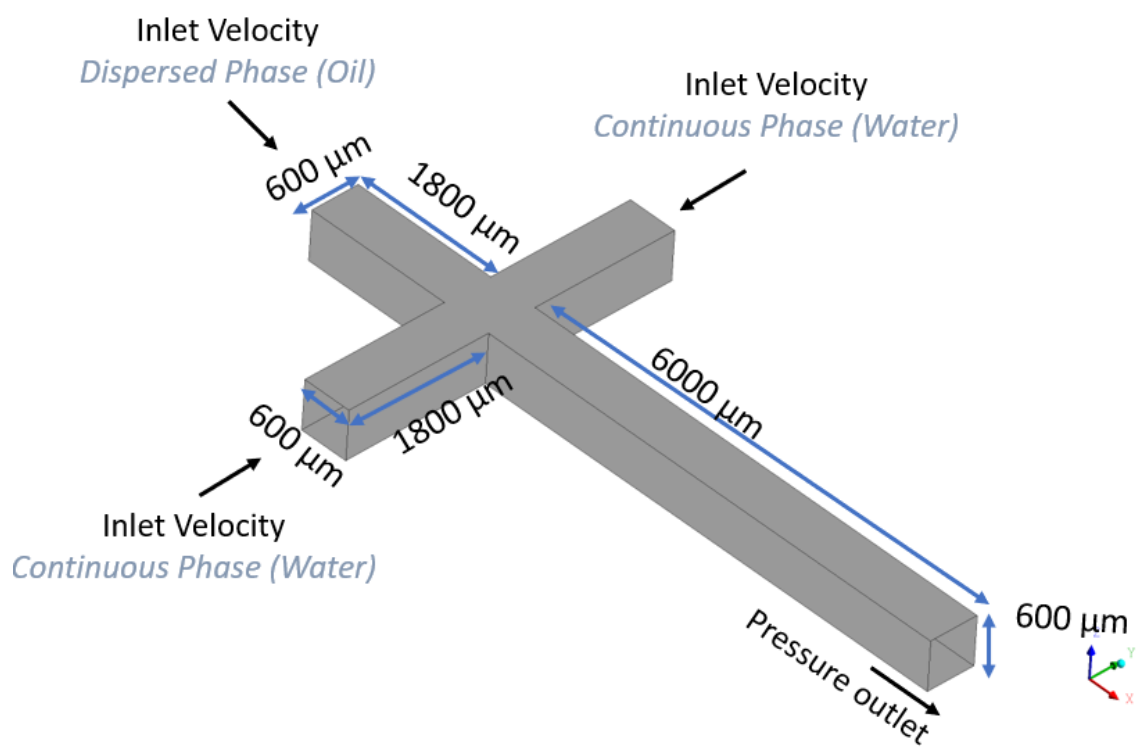

Fig 2. Model geometry, dimensions and boundary conditions for the present verification following Sontti and Atta [19].

A finite volume method based CFD solver from ANSYS Fluent 16 with the implemented Volume of Fluid technique is used to solve the system of time-dependent partial differential equations. For the boundary conditions, constant velocity block profile was utilized for both continuous and dispersed phase inlets. The phase fraction $\alpha$ is set to $\alpha=0$ at the two inlets of the continuous phase and $\alpha=1$ at the inlet of the dispersed phase. The walls of the channels were considered as fully wetted by the continuous phase, therefore the contact angle was set as zero at the walls. No slip boundary conditions are applied at the walls. Pressure boundary was specified at the outlet of the main channel. Table 2 summarizes the varied boundary conditions for each case investigated. The first two cases serve as a verification of our model. All other cases are completely different from [19] and cover a large range of new velocities for the continuous phase. The purpose is to investigate in detail how these velocities influence the flow regimes (dripping (D) or jetting (J)), which will be introduced in the following sections. The inlet velocity of the dispersed phase (oil) was kept constant at $u_{d}=0,0185 \mathrm{~m} / \mathrm{s}$ for all 10 cases. 
Table 2. Boundary conditions varied for the investigation of droplet formation in the present work. In column 1: (D) means dripping regime, (J) means jetting regime.

\begin{tabular}{|c|c|c|c|c|}
\hline $\begin{array}{c}\text { Case } \\
\& \\
\text { regime }\end{array}$ & Continuous phase & $\begin{array}{l}\text { Inlet velocity of the conti- } \\
\text { nuous phase, } u_{c}[\mathrm{~m} / \mathrm{s}]\end{array}$ & $\begin{array}{c}\text { Surface } \\
\text { tension, } \sigma \\
{[\mathrm{mN} / \mathrm{m}]}\end{array}$ & Notes \\
\hline 1 (D) & Water & 0,01850 & 5,37 & Verification \\
\hline $2(\mathrm{D})$ & Water $+40 \%$ glycerol & 0,01850 & 5,04 & Verification \\
\hline $3(\mathrm{D})$ & Water & 0,00925 & 5,37 & New $\mathrm{u}_{\mathrm{c}}$ \\
\hline $4(\mathrm{D})$ & Water $+40 \%$ glycerol & 0,00925 & 5,04 & New $\mathrm{u}_{\mathrm{c}}$ \\
\hline $5(\mathrm{~J})$ & Water & 0,07400 & 5,37 & New $\mathrm{u}_{\mathrm{c}}$ \\
\hline $6(\mathrm{~J})$ & Water $+40 \%$ glycerol & 0,07400 & 5,04 & New $\mathrm{u}_{\mathrm{c}}$ \\
\hline $7(\mathrm{~J})$ & Water & 0,10000 & 5,37 & New $\mathrm{u}_{\mathrm{c}}$ \\
\hline $8(\mathrm{~J})$ & Water $+40 \%$ glycerol & 0,03700 & 5,04 & New $\mathrm{u}_{\mathrm{c}}$ \\
\hline $9(\mathrm{~J})$ & Water & 0,14000 & 5,37 & New $\mathrm{u}_{\mathrm{c}}$ \\
\hline $10(\mathrm{~J})$ & Water $+40 \%$ glycerol & 0,05100 & 5,04 & New $\mathrm{u}_{\mathrm{c}}$ \\
\hline
\end{tabular}

Making use of the mesh sensitivity analysis made by Sontti and Atta [19], we considered a near wall mesh refinement with a first row of thickness of $6 \mu \mathrm{m}$ and continuing with a $13 \%$ size increase towards the middle of the channel. The middle elements reach $35 \mu \mathrm{m}$ length size, and an overall of 390000 elements are required for the hexahedral equidistant mesh. Figure 3 shows a zoomed view of the hexahedral elements in the cross region where the two fluids meet.

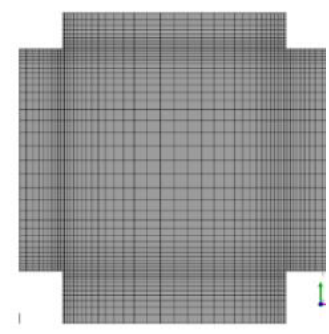

Fig 3. Zoomed view of the hexahedral elements in the cross region where the two fluids meet

A variable time step and a fixed Courant number $(\mathrm{Co}=0,25)$ were considered for solving equations $1-3$.

\section{Results and discussion}

\subsection{Verification and experimental comparison}

As mentioned before, in order to examine the efficiency of the VOF model, first a verification of the results with the work of Sontti and Atta [19] is made. The authors of [19] utilized a combination of the VOF and LV (Level Set) methods, which compared to the pure showed more accurate methodology in capturing the interface between the two fluids. A detailed comparison between the VOF and the CLSVOF models is made by Keshavarzi et al. [21]. As a next step, a visual comparison with the experimental work of Wu et al. [22] was carried out, in order to further confirm the accuracy of the model. 
For our verification, cases 1 and 2 from Table 3 are considered and compared with identical simulations from [19].

Figure 4 shows the comparison of the droplet formation in the middle plane of the domains through time in the two studies for case 1. Both results show very close time scales regarding the generation of oil (blue) in water (red) droplets. The whole process can be divided into three stages. In the first, so-called filling stage $(0,020 \mathrm{~s}-0,040 \mathrm{~s})$, the dispersed phase is injected into the main channel. At some point the growing oil-front blocks the flow from the side channels causing the upstream pressure to increase until it reaches a critical value where the continuous phase begins to squeeze the interface [23]. In the second stage $(0,040 \mathrm{~s}-0,055 \mathrm{~s})$ the dispersed phase (oil) is still being injected into the droplet at a constant flow rate, while the neck starts to collapse. This accelerates the last pinch-off stage, in which droplet detaching occurs (around 0,060s). The three stages described above form, the so-called dripping regime in a microfluidic device [24].
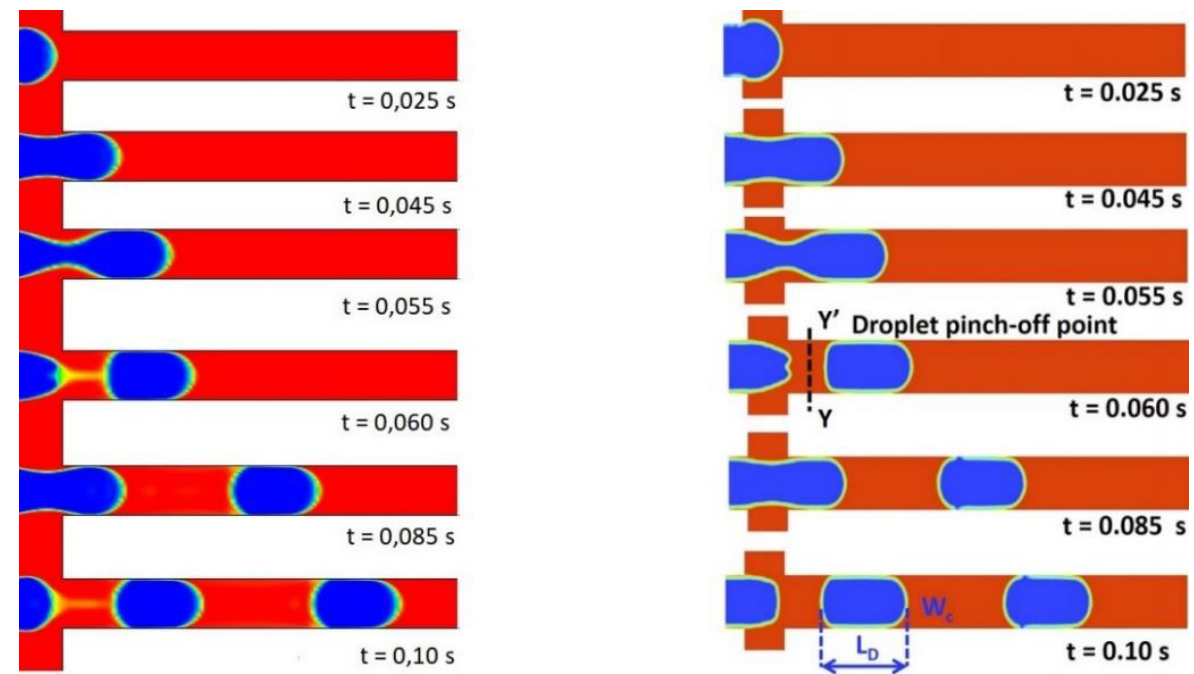

Fig. 4. Comparison of the droplet formation in our work (left) and Sontti and Atta [19] (right) for case 1. Results from [19] are reproduced with permission

Similar observations are made in the second case (case 2 of Table 2), in which water $+40 \%$ glycerol is utilized as continuous phase. The comparison is shown in Fig. 5. For this case the generated droplets have smaller volumes and higher formation frequencies compared to pure water (case 1). At the same operating condition for oil-water $+40 \%$ glycerol system, an earlier droplet breakup $(0,051 \mathrm{~s})$ is also observed. This is due to the higher shear force, 'cutting' the droplet, connected with the higher viscosity of the red colored fluid in the second case. A larger difference in viscosity makes it possible to increase the shear at the boundary between the fluids thus increasing the generation frequencies. 

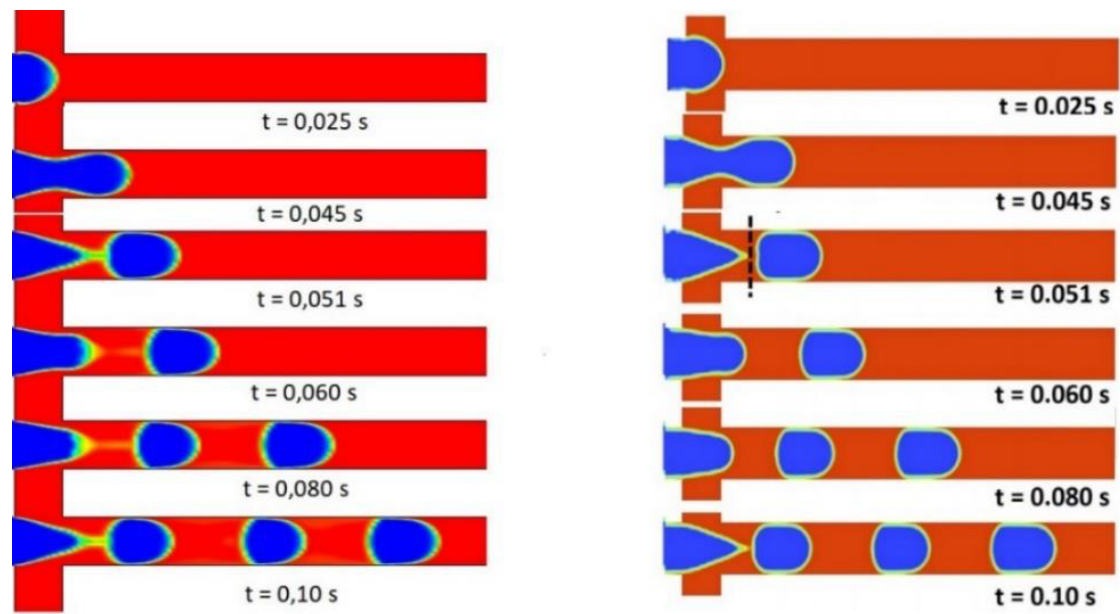

Fig. 5. Comparison of the droplet formation in our work (left) and Sontti and Atta [19] (right) for case 2. Results from [19] are reproduced with permission.

A quantitative comparison of the two cases considered is also carried out and summarized in Table 3. The nondimensional droplet length with respect to channel width $\left(=L_{D} / W c\right)$ and the droplet volume $V_{D}$ are utilized for this purpose. For the calculation of $V_{D}$ Equation 8 is used, with $f$ standing for the frequency of droplet formation in Hertz and $Q_{d}$ being the volume flow rate of the dispersed phase:

$$
V_{D}=\frac{Q_{d}}{f} .
$$

Table 3. Quantitative comparison of the results in our work with the work of Sontti and Atta

\begin{tabular}{|c|c|c|c|c|c|c|}
\hline & \multicolumn{3}{|c|}{ Water / Oil } & \multicolumn{3}{c|}{ Water + 40\% Glycerol / Oil } \\
\hline Parameter & $\begin{array}{c}\text { Our } \\
\text { work }\end{array}$ & $\begin{array}{c}\text { Sontti \& } \\
\text { Atta [19] }\end{array}$ & $\begin{array}{c}\text { Rel. error } \\
{[\%]}\end{array}$ & $\begin{array}{c}\text { Our } \\
\text { work }\end{array}$ & $\begin{array}{c}\text { Sontti \& } \\
\text { Atta [19] }\end{array}$ & $\begin{array}{c}\text { Rel. error } \\
{[\%]}\end{array}$ \\
\hline $\mathbf{L}$ [/W & 1,92 & 2,12 & 9,5 & 1,34 & 1,46 & 8,8 \\
\hline $\begin{array}{c}\text { Droplet } \\
\text { Volume [nL] }\end{array}$ & 257 & 298 & 13,6 & 152 & 175 & 14,2 \\
\hline
\end{tabular}

A relative difference of around $13-14 \%$ is observed for the volume of the generated droplets and around $10 \%$ for their length with respect to the channel width. This is in a reasonably good agreement with the CLSVOF method presented in [19], as the VOF technique often tends to smear the step profile of the interface over several mesh cells because of numerical diffusion, as it has been demonstrated in [22].

To further confirm the accuracy of the utilized VOF method, an additional comparison with the experimental work of Wu et al [22] was carried out. Numerical simulations were performed with the same fluid properties and channel dimensions, considered by the mentioned authors. Figure 6 demonstrates the visual comparison of droplet development. Here, the velocity of the continuous phase is $u_{c}=0,00252 \mathrm{~m} / \mathrm{s}$, the velocity of the discrete phase $-u_{d}=0,00084 \mathrm{~m} / \mathrm{s}$ and the surface tension is $\sigma=30 \mathrm{mN} / \mathrm{m}$. 


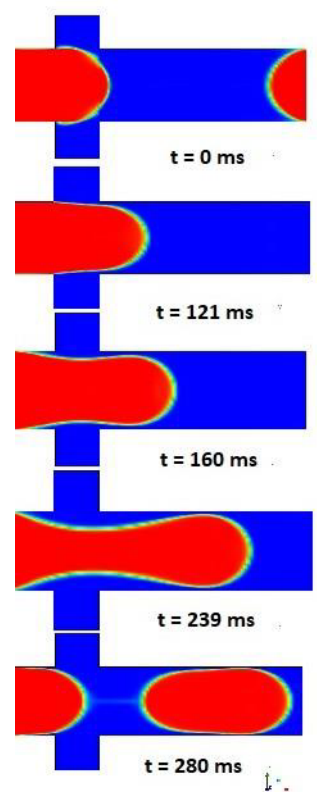

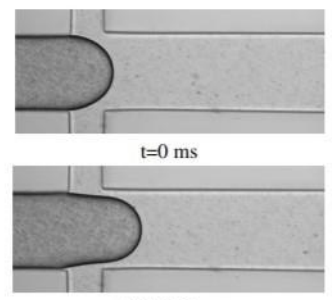

$\mathrm{t}=121.159 \mathrm{~ms}$

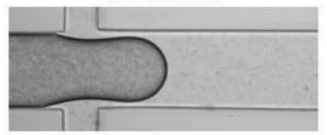

$\mathrm{t}=160.528 \mathrm{~ms}$

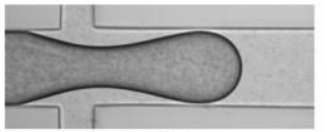

$\mathrm{t}=239.126 \mathrm{~ms}$

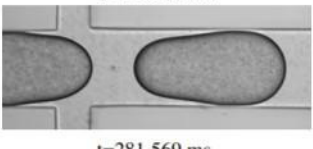

$t=281569 \mathrm{~ms}$

Fig. 6. Comparison of the droplet formation in our work (left) and the experimental work of $\mathrm{Wu}$ et al. [22] (right) Reproduced with permission.

\subsection{Influence of continuous phase velocity and significance of the capillary number for the transition of regimes}

In order to understand better how droplet size for the flow-focusing microdroplet generator could be controlled more precisely, eight additional cases with varied continuous phase velocities (at constant dispersed flow rate of $u_{d}=0,0185 \mathrm{~m} / \mathrm{s}$ ) were carried out, extending the range of simulated values by Sontti and Atta [19]. First, the value of $u_{c}$ was decreased two times, compared to cases 1 and 2, to further evaluate the characteristics of the drops in the dripping regime (cases 3 and 4 from Table 2). Figure 7(a) shows the pressure variation along the center line of the channel for these two cases. Due to the increase in the internal friction of the continuous phase in case 4, a bigger total pressure in the domain (around $40 \%$ ) is observed when compared to case 3 . In any pressure profile, each peak and its width indicate a droplet position and shape, respectively. Due to their smaller size and hence, larger surface curvatures, the droplets from case 4 have on average approx. $15 \%$ higher pressure differences with their surrounding compared to case 3 . 


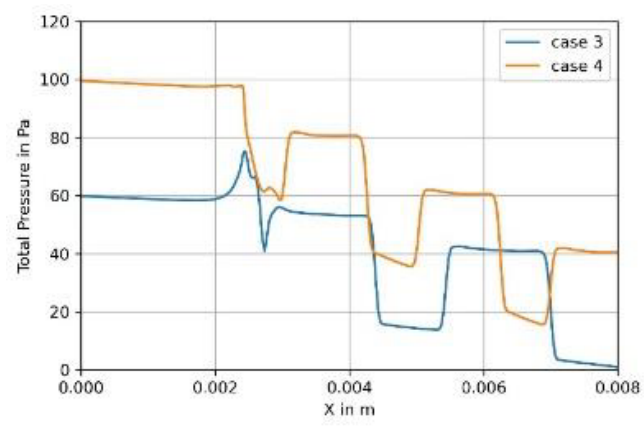

(a)

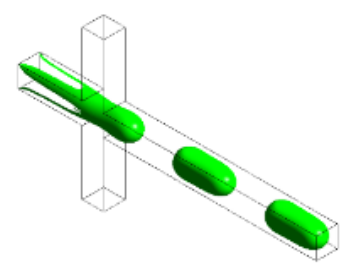

(b)

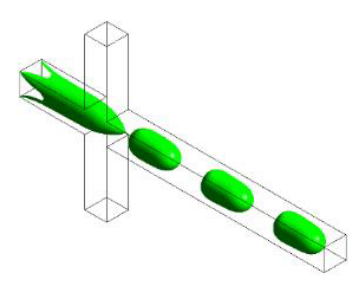

(c)

Fig. 7 Comparison of case 3 and 4 from Table 2: (a) the total pressure profiles along the channel centerline ; (b) a three dimensional iso-surface evolution of droplet shapes for case 3 ; (c) a three dimensional iso-surface evolution of droplet shapes for case 4

Similar to cases 1 and 2, the droplet length in cases 3 and 4 decreases with increasing continuous phase viscosity, when comparing the three-dimensional iso-surface evolutions. As seen in Figure 7(b) and Figure 7(c) both cases are again in the dripping flow regime, described in section 3.1 .

In the next step the velocity of the continuous phase is increased four times, compared to cases 1 and 2 (again for $u_{d}=0,0185 \mathrm{~m} / \mathrm{s}$ ). This way we were able to further investigate the effect of $u_{c}$ on the droplets and answer the question if the dripping regime take up even at higher velocity values. Compared to Figure 7, even larger differences in the total pressure can be seen between cases 5 and 6; these are shown in Figure 8(a). The bigger curvature radii lead to bigger resulting capillarity forces and hence, to three times higher pressure differences between the inside and the outside of the droplets as compared to cases 3 and 4. A noticeable pressure gradient across the droplet itself can also be seen. This is due to the difference in radius of curvature from back to nose of a droplet, shown also in the theoretical analysis made by Abiev et al. [25]. From Figures 8(b) and 8(c) a clear change in the shape and size of droplets is visible. Due to the increased "cutting" shear force on the dispersed phase, droplet length and volume for case 6 (when compared to case 5) decrease and higher formation frequencies occur. In this, so called jetting regime, a longsuspended column of the disperse fluid flows in the central channel as shown in Figures 8 (b) and 8(c). The breakup of drops does not occur due to shear stress but due to the pressure drop across the emerging drop [26]. 


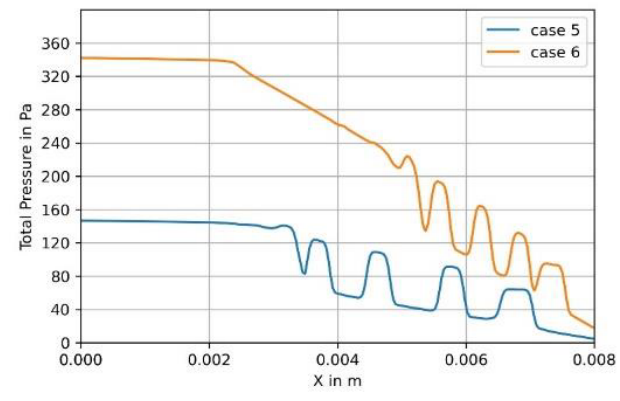

(a)

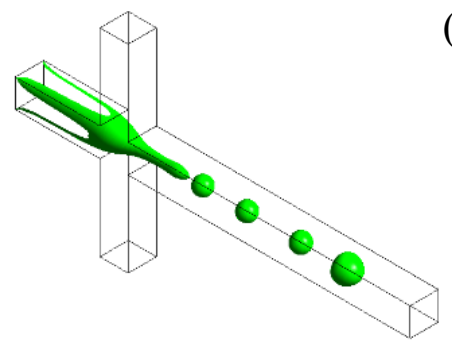

(b)

(c)

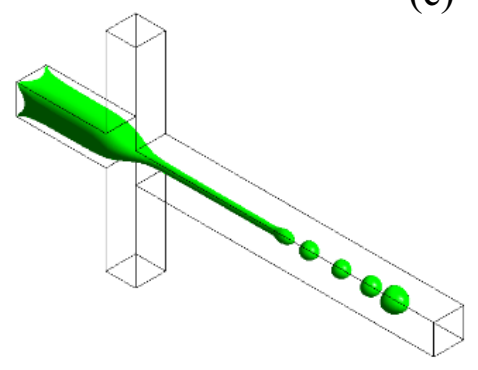

Fig. 8 Comparison of case 5 and 6 from Table 2: (a) the total pressure profiles along the channel centerline ; (b) a three dimensional iso-surface evolution of droplet shapes for case 5; (c) a three dimensional iso-surface evolution of droplet shapes for case 6

The question where the transition from the dripping to the jetting regimes occurs has been discussed in the literature [27]. One of the critical parameters thoroughly investigated in droplet producing microfluidic devices is the capillary number, described in Equation 9:

$$
C a=\frac{u_{c} \mu_{c}}{\sigma}
$$

where $\mu_{c}$ is the dynamic viscosity of the continuous phase. The number identifies the ratio of viscous to interfacial forces. For low capillary numbers, capillary forces dominate and therefore big droplets will be formed, whereas for high capillary numbers viscous forces generate small drops, increasing their surface energy. Similar to the Reynolds $(R e)$ number, the $\mathrm{Ca}$ number can be used to determine a transition point between two flow regimes. One way to do this is to show the geometrical changes in the generated droplets as a function of $\mathrm{Ca}$. Figure 9 shows the non-dimensional length of the droplets for the 10 cases as a function of the Capillary number. In the Figure it can be seen that the largest changes of the drop size occur up to $C a \approx 0,02$. The Figure shows that for even larger $C a$ numbers $(C a>0,03)$ the droplet size stagnates, i.e. it does not change significantly. Such a qualitative behavior is observed also in other studies with other geometries (e.g. Figure 6 from [28]).

At the left-hand side of the figure, the size of the droplets increases significantly when the $\mathrm{Ca}$ value decreases under the value 0,01 .

Figure 9 allows clarifying the position of the transition point between the two regimes. The flow change appears where the droplet length $L$ becomes smaller than the channel width 
$W$. For water (blue) this value is around $C a \approx 0,015$ and for water $+40 \%$ glycerol (red) it is $C a \approx 0,025$. The blue and red symbols show the same qualitative trend: larger slope for smaller $C a$ numbers and an almost constant droplet size for larger $C a$ numbers. The difference in the transition points of the two fluids is attributed to the different fluid properties. Although the $\mathrm{Ca}$ number of the continuous phase is the most important parameter that indicates the transition, there are other properties (viscosity of the dispersed phase, surface tension) which also influence the flow regimes.

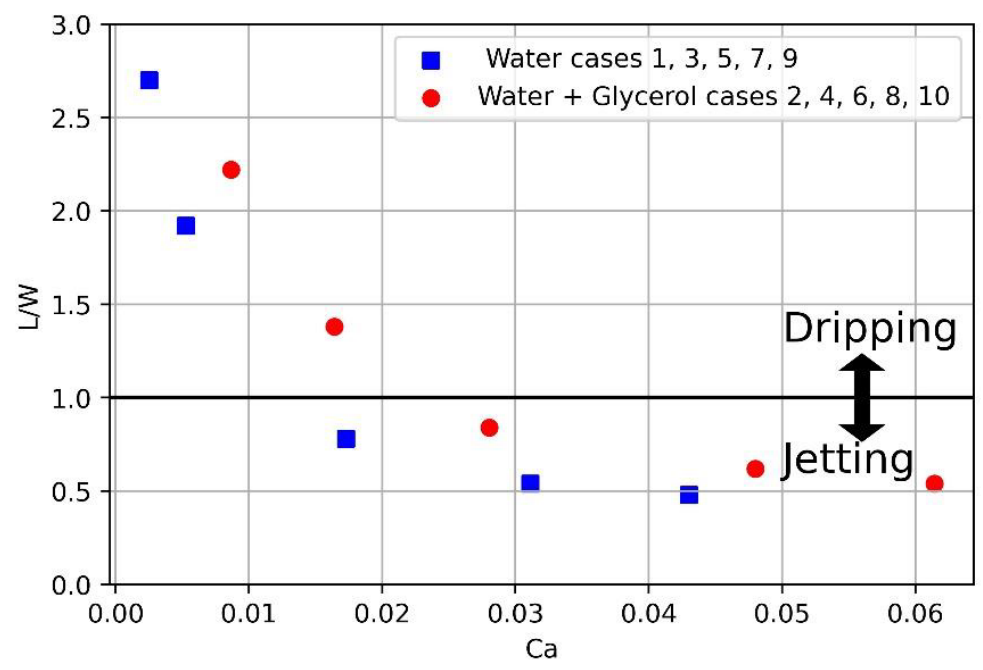

Fig. 9 The nondimensional droplet length with respect to channel width as a function of $\mathrm{Ca}$

It is important to note that for the definition of the Ca number, the average domain velocity - and not the inlet velocity - of the continuous phase was utilized. Table 4 summarizes the averaged domain values for the continuous phase which are used in Equation 9 and Fig. 9.

Table 4 Average domain velocities for the 10 cases, utilized to calculate the Ca number

\begin{tabular}{|c|c|}
\hline Case & Average $\boldsymbol{u}_{\boldsymbol{c}}$ in the domain \\
\hline 1 & 0,031 \\
\hline 2 & 0,025 \\
\hline 3 & 0,015 \\
\hline 4 & 0,013 \\
\hline 5 & 0,104 \\
\hline 6 & 0,093 \\
\hline 7 & 0,187 \\
\hline 8 & 0,042 \\
\hline 9 & 0,259 \\
\hline 10 & 0,072 \\
\hline
\end{tabular}




\section{Conclusion}

In this work the droplet generation in a flow-focusing microfluidic device has been investigated. The continuous phase (water, or water $+40 \%$ glycerol) was introduced through the two side channels and the dispersed phase (oil: octane $+2,5 \%$ SPAN 80 ) was entered from the main channel. For all simulations the VOF method was utilized. In the first part, a verification of the technique with the results from Sontti and Atta [19] has been made. Here, two cases were simulated, in the first, pure water was used as a continuous phase, and in the second - water $+40 \%$ glycerol which leads to increased viscosity. A good agreement between the two works, with maximum differences of $13 \%$ is found which are attributed mainly to the different numerical techniques. To further confirm the accuracy of the utilized VOF method, an additional comparison with the experimental work of $\mathrm{Wu}$ et al [22] was carried out which shows a very good agreement.

In the second part of the presented study, the influence of velocities of the continuous phase on the droplet formation is investigated. Two main flow regimes are observed dripping and jetting, which are distinguished by the size of the droplet (for jetting, the droplets are smaller than the width of the channel). The transition between these regimes is investigated. This is done for a wide range of velocities $u_{c}\left(0,1400 \mathrm{~m} / \mathrm{s}>u_{c}>0,00925 \mathrm{~m} / \mathrm{s}\right)$. The large number of studied regimes enabled the following main observations for the investigated setup:

- At very large velocities $u_{c}(\mathrm{Ca}>0,03)$ droplet size reaches a point of saturation, hence droplets do not decrease their size with increasing velocity $u_{\mathrm{c}}$ any more.

- At very small velocities $u_{c}$, $(C a<0,01)$ the observation is that droplet size still increases strongly with decreasing velocity.

- There is a critical $C a$ number, which serves as a transition point between the two described flow regimes (dripping and jetting). For the cases with water as a continuous phase, a critical value around $C a \approx 0,015$ is observed, whereas for the cases with water $+40 \%$ glycerol it is $C a \approx 0,025$. This difference is due to the influence of the dispersed phase properties.

- It was found that at higher flow rates (velocities) higher total pressures and pressure jumps in the droplets occur, also leading to the formation of smaller drops. The same effect was observed with increasing continuous phase viscosity.

As a whole, the present study shows that the VOF method is a reliable technique for the simulation and prediction of droplet generation in flow-focusing channels. It will allow the future study of other diverse setups and of various fluid combinations.

\section{References}

1. N. Nguyen, S. Wereley, Fundamentals and Applications of Microfluidics; Artech House Publishing: Norwood, MA, USA, (2007)

2. E. Grigorov, B. Kirov, M.B. Marinov, V. Galabov, Micromachines 12, 498 (2021)

3. Y. Xu, Z. Zhang, Z. Zhou, X. Han, X. Liu, Micromachines, 11, 187 (2020)

4. B.K. Madhusudan, G. Sanket, Eng. Res. Express 2, 042001 (2020)

5. N. Shembekar, C. Chaipan, R. Utharala, C. A. Merten, Lab Chip, 16, 1314-1331 (2016)

6. T. Thorsen, R. W. Roberts, F. H. Arnold, S. R. Quake ,Phys. Rev. Lett., 86, 4163 4166 (2001)

7. W. Zeng, H. Fu, IEEE 8th International Conference on Fluid Power and Mechatronics (FPM), 1190-1196 (2019) 
8. S. L. Anna, N. Bontoux, H. A. Stone, Appl. Phys. Lett., 82, $364-366$ (2003)

9. L. Yobas ,S. Martens, W. L. Ong, N. Ranganathan, Lab Chip, 6, 1073 -1079 (2006)

10. C. Cramer, P. Fischer, E. J. Windhab Chem. Eng. Sci., 59, 3045 -3058 (2004)

11. L. Mazutis, J. Gilbert, W. Ung, Nat Protoc 8, 870-891 (2013).

12. M. A. Bijarchi, A. Favakeh, S. Alborzi, M. B. Shafii, Sensors and Actuators B: Chemical, 329, (2021)

13. Y. Zhang, L. Wang, Nanoscale and Microscale Thermophysical Engineering, 13:4, 228-242 (2009)

14. W. Lan, S. Li, G. Luo, Chem.Eng. Sc., 134, 76-85, (2015)

15. Y. Lu, T. Fu, C. Zhu, Y. Ma, H. Z. Li, Chem. Eng. Sc., 105, 213-219, (2014)

16. W. Lan, S. Li, Y. Wang, G. Luo, Ind. Eng. Chem. Res., 53, 4913 (2014)

17. Y. Li, M. Jain, Y. Ma, Nandakumar, Soft Matter, 11, 3884 (2015)

18. A. Gupta, H. S. Matharoo, D. Makkar, R. Kumar, Comput. Fluids, 100, 218 (2018)

19. S. Sontti, A. Atta, Ind. Eng. Chem. Res 59, 3702-3716 (2020)

20. C. Yao, Y. Liu, C. Xu, S. Zhao, G. Chen, AIChE J., 64, 346 (2018)

21. G. Keshavarzi, G.H. Yeoh, T. Barber, The Journal of Comput. Mult. Flows. 5(1), 4355 (2013)

22. L. Wu, M. Tsutahara, L. S. Kim, M. Ha, Int. J. Multiphase Flow, 34, 852 (2008)

23. P.A. Romero, A.R. Abate, Lab Chip, 12, 5130-5132 (2012)

24. X. Chen, T. Glawdel, N. Cui, Microfluid Nanofluid 18, 1341-1353 (2015).

25. R.Sh. Abiev, Chemical Engineering Science, Volume 174, 403-412 (2017)

26. N.M. Kovalchuk, M. Sagisaka, K. Steponavicius, Microfluid Nanofluid, 23, 103 (2019).

27. X. Chen, T. Glawdel, N. Cui, Microfluid Nanofluid 18, 1341-1353 (2015).

28. S.K. Moon, I.W. Cheong, S.W. Choi, Colloids and Surfaces A: Phys. And Eng. Aspects, 454, 84-88 (2014) 